PROCEEDINGS OF THE

AMERICAN MATHEMATICAL SOCIETY

Volume 125, Number 2, February 1997, Pages 387-392

S 0002-9939(97)03703-9

\title{
IDEALS CONTRACTED FROM 1-DIMENSIONAL OVERRINGS WITH AN APPLICATION TO THE PRIMARY DECOMPOSITION OF IDEALS
}

\author{
WILLIAM HEINZER AND IRENA SWANSON
}

(Communicated by Wolmer V. Vasconcelos)

\begin{abstract}
We prove that each ideal of a locally formally equidimensional analytically unramified Noetherian integral domain is the contraction of an ideal of a one-dimensional semilocal birational extension domain. We give an application to a problem concerning the primary decomposition of powers of ideals in Noetherian rings. It is shown in an earlier paper by the second author that for each ideal $I$ in a Noetherian commutative ring $R$ there exists a positive integer $k$ such that, for all $n \geq 1$, there exists a primary decomposition $I^{n}=Q_{1} \cap \cdots \cap Q_{s}$ where each $Q_{i}$ contains the $n k$-th power of its radical. We give an alternate proof of this result in the special case where $R$ is locally at each prime ideal formally equidimensional and analytically unramified.
\end{abstract}

In this paper we prove that every ideal in a locally formally equidimensional analytically unramified Noetherian ring $R$ is the contraction of an ideal of a onedimensional semilocal extension which is essentially of finite type over $R$. If $R$ is a domain, the extension may be taken to be birational, i.e., with the same field of fractions as $R$.

By passing to the extended Rees ring $R\left[I t, t^{-1}\right]$ of an ideal $I$ of $R$, these contraction properties give a type of uniform primary decomposition for the powers of $I$. This is based on the fact that the primary decomposition of a height-one ideal in a one-dimensional semilocal ring is unique, and the primary decomposition for powers of a fixed ideal in such a ring is obtained from just taking the powers of the primary components of the fixed ideal. Furthermore, contracting primary decompositions from an overring gives a primary decomposition for the contracted ideal. Our interest in establishing this result was motivated by a question, recently answered in [S2], concerning the primary decompositions of powers of an ideal.

All rings we consider are commutative and our notation is as in $[\mathrm{AM}]$ and $[\mathrm{M}]$.

\section{POWERS OF IDEALS AND PRIMARY DECOMPOSITIONS}

Let $I$ be a proper ideal of a commutative Noetherian ring $R$. It is known that only finitely many prime ideals of $R$ are associated primes of a power of $I$ [Rat], and that all suitably large powers of $I$ have the same associated primes [B]. In considering primary decompositions of the powers of $I$, it is natural to ask about the growth of the exponents of primary components of $I^{n}$, where the exponent of

Received by the editors September 6, 1995.

1991 Mathematics Subject Classification. Primary 13C05, 13E05, 13 H99.

The authors thank Craig Huneke for helpful suggestions concerning this paper. 
a primary ideal $Q$ with $\operatorname{rad}(Q)=P$ is the smallest positive integer $e$ such that $P^{e} \subseteq Q$ [ZS, page 153]. If $Q$ is a primary component associated to a minimal prime $P$ of $I$, then $Q^{(n)}$, the inverse image in $R$ of $Q^{n} R_{P}$, is the unique $P$-primary component of $I^{n}$ and the exponent growth of the $P$-primary component of $I^{n}$ is linearly bounded as a function of $n$ in the sense that if $Q$ has exponent $e$, then $P^{e n} \subseteq Q^{(n)}$. The situation, however, for embedded associated primes of $I$ is not as obvious [He], [S1]. By proving a version of the linear uniform Artin-Rees lemma in the spirit of Huneke's paper [Hu], it is shown in [S2] that there exist primary decompositions of the powers $I^{n}$ of $I$ for which the exponent growth of the primary components is linearly bounded. We present here an alternative approach to obtain a special case of this result.

If $I=Q_{1} \cap \cdots \cap Q_{s}$ is a primary decomposition, then we clearly have

$$
I^{n} \subseteq Q_{1}^{n} \cap \cdots \cap Q_{s}^{n},
$$

but in general the inclusion in (1.1) may be proper, and powers of a primary ideal need not be primary.

A case where equality holds in (1.1) is if the intersection of the $Q_{i}$ is also their product. And a case where the intersection of ideals is their product is that of pairwise comaximal ideals. Thus if $\operatorname{dim}(R / I)=0$, then the primary components of $I$ are pairwise comaximal and for each positive integer $n, I^{n}=Q_{1}^{n} \cap \cdots \cap Q_{s}^{n}$ is the unique irredundant primary decomposition of $I^{n}$. Our proof of a special case of the linearly bounded exponent growth result of [S2] is based on obtaining primary decompositions for the powers of $I$ via descent from a regular principal ideal of a one-dimensional semilocal extension ring.

We use the following elementary lemma.

Lemma 1.2. Suppose $R$ is a subring of a ring $S$ and $x \in R$ is a regular element of $S$. If $x R=x S \cap R$, then $x^{n} R=x^{n} S \cap R$ for each positive integer $n$.

Proof. We clearly have $x^{n} R \subseteq x^{n} S \cap R$. Assume by induction that $n \geq 2$ and $x^{n-1} R=x^{n-1} S \cap R$. Then

$$
x^{n} S \cap R=x^{n} S \cap x R=x\left(x^{n-1} S \cap R\right)=x x^{n-1} R=x^{n} R,
$$

where equality in the middle step uses that $x$ is a regular element of $S$.

Remark 1.3. With $R, S, x$ as in (1.2), if $S$ is one-dimensional and Noetherian, then each associated prime of $x S$ is a maximal ideal of $S$ and a minimal prime of $x S$. Hence the ideal $x S$ has a unique irredundant primary decomposition, say $x S=$ $Q_{1} \cap \cdots \cap Q_{s}$, and for each positive integer $n, x^{n} S=Q_{1}^{n} \cap \cdots \cap Q_{s}^{n}$ is the unique irredundant primary decomposition of $x^{n} S$. If $x R=x S \cap R$, then by (1.2), we have

$$
x^{n} R=\left(Q_{1}^{n} \cap R\right) \cap \cdots \cap\left(Q_{s}^{n} \cap R\right)
$$

for each positive integer $n$. Since $Q_{i}^{n}$ is primary in $S$, the ideal $Q_{i}^{n} \cap R$ is primary in $R$. The decomposition given in (1.4) may fail to be irredundant, but it can be shortened to an irredundant primary decomposition. Moreover, if $\operatorname{rad}\left(Q_{i}\right)=M_{i}$ and $e_{i}$ is the exponent of $Q_{i}$, then for $k=\max \left\{e_{1}, \ldots, e_{s}\right\}$ we have $M_{i}^{k n} \subseteq Q_{i}^{n}$ for each $i$, and therefore $\left(M_{i} \cap R\right)^{k n} \subseteq\left(Q_{i}^{n} \cap R\right)$ for each $i$. This shows that the exponent growth of the primary components of $x^{n} R$ in a primary decomposition obtained from (1.4) is linearly bounded. 
Remark 1.5. Let $I$ be an ideal of a Noetherian ring $R$ and let $t$ be an indeterminate over $R$. With $S=R\left[I t, t^{-1}\right]$, the extended Rees ring of $I$, we clearly have $t^{-n} S \cap R=I^{n}$ for each positive integer $n$. Therefore to show the existence of primary decompositions of the powers of $I$ with linearly bounded exponent growth, by passing from $I$ to the principal ideal $t^{-1} S$, it suffices to consider the case where $I$ is a principal ideal generated by a regular element.

In view of (1.3) and (1.5), we are led to ask:

Question 1.6. Suppose $R$ is a Noetherian ring and $x \in R$ is a regular element. Does there exist a one-dimensional Noetherian extension ring $S$ of $R$ such that $x$ is a regular element of $S$ and $x R=x S \cap R$ ?

In $\S 2$ we present an affirmative answer to (1.6) for a restricted class of Noetherian rings by proving that ideals in this restricted class of rings contract from onedimensional Noetherian ring extensions. We are aware of no example where (1.6) has a negative answer.

\section{OnE-DIMENSIONAL SEMILOCAL EXTENSION RINGS}

Let $R$ be a Noetherian ring and let $I$ be an ideal in $R$. We prove in this section that under certain assumptions on $R, I$ contracts from a one-dimensional semilocal Noetherian ring extension. Let $I=Q_{1} \cap \cdots \cap Q_{s}$ be an irredundant primary decomposition and let $P_{i}=\operatorname{rad}\left(Q_{i}\right)$. Our first step is to prove that each $Q_{i} R_{P_{i}}$ contracts from a Noetherian ring extension of $R_{P_{i}}$ which has smaller dimension than $R_{P_{i}}$ (see Theorem 2.1 for the precise statement). This and induction on dimension then imply that each $Q_{i} R_{P_{i}}$, and hence also $Q_{i}$, is contracted from a one-dimensional Noetherian ring extension. Theorems 2.3 and 2.4 then prove the contraction property for all ideals $I$ in locally formally equidimensional analytically unramified Noetherian rings. Corollary 2.6 then gives the linear growth of exponents of primary components of powers of an ideal.

Theorem 2.1. Let $(R, \mathbf{m})$ be a reduced local ring and let $Q$ be an $\mathbf{m}$-primary ideal. Assume that the integral closure $R^{\prime}$ of $R$ in its total quotient ring is a finitely generated $R$-module and that the height of each maximal ideal of $R^{\prime}$ is at least two. Then there exist regular elements $a, b \in \mathbf{m}$ such that $\mathbf{m} R[a / b]$ is a nonmaximal prime ideal of $R[a / b]$, and $S=R[a / b]_{\mathbf{m} R[a / b]}$ is a local ring with $\operatorname{dim}(S)<\operatorname{dim}(R)$ and $Q S \cap R=Q$.

Proof. Since $R$ is reduced, the total quotient ring of $R$ is a finite product of fields and $R^{\prime}$ is a finite product of normal Noetherian domains, say $R^{\prime}=R_{1}^{\prime} \times \cdots \times R_{m}^{\prime}$. Let $r$ be a positive integer such that $\mathbf{m}^{r} \subseteq Q$. By the Artin-Rees lemma, there exists a positive integer $n$ such that $\mathbf{m}^{n} R^{\prime} \cap R \subseteq \mathbf{m}^{r}$. Let $a, b \in \mathbf{m}^{n}$ be such that the ideal $(a, b) R^{\prime}$ has height two. It follows that $a$ and $b$ are regular elements of $R$ and the images of $a, b$ in $R_{i}^{\prime}$ form a regular sequence for $1 \leq i \leq m$. Let $t$ be an indeterminate over $R^{\prime}$ and let $\phi^{\prime}: R^{\prime}[t] \rightarrow R^{\prime}[a / b]$ be the $R^{\prime}$-algebra homomorphism such that $\phi^{\prime}(t)=a / b$. Then $R^{\prime}[t]=R_{1}^{\prime}[t] \times \cdots \times R_{m}^{\prime}[t]$. Since the images of $a, b$ in each $R_{i}^{\prime}$ form a regular sequence, $\operatorname{ker}\left(\phi^{\prime}\right)=(b t-a) R^{\prime}[t]$. Let $\phi: R[t] \rightarrow R[a / b]$ be the restriction of $\phi^{\prime}$. Since $\operatorname{ker}\left(\phi^{\prime}\right) \subset \mathbf{m}^{n} R^{\prime}[t]$ and $\operatorname{ker}(\phi)=\operatorname{ker}\left(\phi^{\prime}\right) \cap R[t], \operatorname{ker}(\phi) \subset \mathbf{m}^{r} R[t]$. Since $\mathbf{m} R[t]$ is a nonmaximal prime ideal of $R[t]$ with $\mathrm{ht}(\mathbf{m})=\mathrm{ht}(\mathbf{m} R[t])$, and since $Q R[t]$ is $\mathbf{m} R[t]$-primary and $\operatorname{ker}(\phi) \subset Q R[t]$, it follows that $\mathbf{m} R[a / b]$ is a nonmaximal prime ideal and $Q R[a / b]$ is $\mathbf{m} R[a / b]$-primary. Therefore $S=R[a / b]_{\mathbf{m} R[a / b]}$ is a local ring with $\operatorname{dim}(S)<\operatorname{dim}(R)$ and $Q S \cap R=Q$. 
Corollary 2.2. Let $(R, \mathbf{m})$ be a formally equidimensional analytically unramified local ring with $\operatorname{dim}(R)=d \geq 1$, and let $Q$ be an $\mathbf{m}$-primary ideal. There exists a one-dimensional local extension ring $T$ of $R$ such that $T$ is a subring of the total quotient ring of $R$ and is essentially of finite type over $R$, and is such that $Q$ contracts from $T .^{1}$

Proof. The fact that $R$ is analytically unramified implies that the integral closure $R^{\prime}$ of $R$ in its total quotient ring is a finitely generated $R$-module, and that finitely generated $R$-subalgebras of the the total quotient ring of $R$ also have this property [R1, Theorem 1.5]. The assumption that $R$ is formally equidimensional implies that: (i) $R$ is universally catenary, (ii) equidimensional local rings essentially of finite type over $R$ are formally equidimensional, and (iii) all the maximal ideal of $R^{\prime}$ have height equal to $\operatorname{dim}(R)=d$ [M, Theorem 31.6]. If $d>1$, then (2.2) implies the existence of regular elements $a, b \in \mathbf{m}$ such that $\mathbf{m} R[a / b]$ is a nonmaximal prime ideal and $S=R[a / b]_{\mathrm{m} R[a / b]}$ is a local ring with $Q S \cap R=Q$. Since $R$ is equidimensional and universally catenary, $\operatorname{dim}(S)=d-1$, and $S$ is equidimensional, and therefore formally equidimensional. A simple induction argument implies the existence of a one-dimensional local extension $T$ of $R$ such that $T$ is essentially of finite type over $R$, a subring of the total quotient ring of $R$, and $Q T \cap R=Q$.

Now let again $I=Q_{1} \cap \cdots \cap Q_{s}$ be a primary decomposition of $I$ and let $P_{i}=\operatorname{rad}\left(Q_{i}\right)$. By $(2.2)$ we know that each $Q_{i}$ contracts from a one-dimensional local extension ring as long as $R_{P_{i}}$ is formally equidimensional and analytically unramified. The following lemma proves that in this case then $I$ is also contracted from a one-dimensional extension ring.

Lemma 2.3. With notation as above, assume there exists, for each $i, 1 \leq i \leq s$, a one-dimensional local extension ring $T_{i}$ of $R_{P_{i}}$ such that $Q_{i} R_{P_{i}}=Q_{i} T_{i} \cap R_{P_{i}}$. Let $T$ be the direct product $T_{1} \times \cdots \times T_{s}$. Then $T$ is a one-dimensional semilocal extension ring of $R$ and $I$ contracts from $T$, i.e., $I=I T \cap R$.

Proof. Since the canonical map of $R$ into the direct product $R_{P_{1}} \times \cdots \times R_{P_{s}}$ is an injection, and $R_{P_{i}}$ is a subring of $T_{i}$ for $1 \leq i \leq s$, the canonical map of $R$ into $T$ is an injection. It is clear that $T$ is one-dimensional and semilocal. Since $Q_{i}$ is primary it is the inverse image in $R$ of $Q_{i} R_{P_{i}}$. Therefore $Q_{i} T \cap R=Q_{i}$ for $1 \leq i \leq s$. Hence

$$
I T \cap R \subseteq\left(Q_{1} T \cap R\right) \cap \cdots \cap\left(Q_{s} T \cap R\right)=Q_{1} \cap \cdots \cap Q_{s}=I .
$$

Thus every ideal in a locally analytically unramified and formally equidimensional Noetherian ring is contracted from a one-dimensional Noetherian ring extension which is essentially of finite type. In case $R$ is an integral domain one can take the extension to be a domain by replacing the finite direct product in the preceding proof with an intersection. Theorem 2.4 is related to [GH, (3.21)] which applies to a Cohen-Macaulay domain.

\footnotetext{
${ }^{1}$ An alternative proof of this corollary can be given using work of Rees. For simplicity let $(R, \mathbf{m})$ be a reduced equidimensional complete local ring, and let $Q$ be an $\mathbf{m}$-primary ideal. There exists an ideal $I$, generated by parameters, such that the integral closure of $I$ is contained in $Q[\mathrm{R} 1]$. By [R2], it follows that the equations defining the Rees algebra $R[I t]$ have coefficients contained in $Q$, and it then follows that a suitable affine piece of the blowup of $I$, localized at the extension of the maximal ideal $\mathbf{m}$, satisfies the conclusion of (2.2).
} 
Theorem 2.4. Let I be an ideal of a Noetherian integral domain $R$. Assume that for each $P \in \operatorname{Ass}(R / I)$ the local ring $R_{P}$ is analytically unramified and formally equidimensional. Then there exists a one-dimensional semilocal birational extension $T$ of $R$ such that $T$ is essentially of finite type over $R$ and $I T \cap R=I$.

Proof. Let $\operatorname{Ass}(R / I)=\left\{P_{i}\right\}_{i=1}^{s}$, and let $Q_{i}$ be a $P_{i}$-primary component of $I$. By (2.2) there exists a one-dimensional local extension domain $T_{i}$ of $R_{P_{i}}$ such that $T_{i}$ is a subring of the fraction field of $R$ and $Q_{i} T_{i} \cap R_{P_{i}}=Q_{i} R_{P_{i}}, 1 \leq i \leq s$. Since $T_{i}$ has center $P_{i}$ on $R$, for $i \neq j$, the one-dimensional local domains $T_{i}$ and $T_{j}$ are not dominated by a common valuation domain. Hence by [HO, (2.9) and (2.10)], $T=\cap_{i=1}^{s} T_{i}$ is a one-dimensional semilocal domain and each localization of $T$ at a prime ideal is essentially of finite type over $R$. It follows that $T$ is essentially of finite type over $R$, and $Q_{i} T \cap R=Q_{i}$ for each $i, 1 \leq i \leq s$, so $I T \cap R=I$.

As a consequence of these results on contractions of ideals we obtain our results on exponents of primary components of powers of ideals:

Theorem 2.5. Let $R$ be a Noetherian ring and let $x \in R$ be a regular element. Assume that for each associated prime $P$ of $I=x R$, the local ring $R_{P}$ is analytically unramified and formally equidimensional. Then there exists a positive integer $k$ such that, for all $n \geq 1$, there exists a primary decomposition $I^{n}=Q_{1} \cap \cdots \cap Q_{s}$ where each $Q_{i}$ contains the $n k$-th power of its radical.

Proof. By (1.3), it suffices to show the existence of a one-dimensional semilocal extension ring $S$ of $R$ such that $x$ is a regular element of $S$ and $x R=x S \cap R$. This follows by (2.2) and (2.3).

Since the passage from a Noetherian ring to an extended Rees ring preserves the property of being locally formally equidimensional and analytically unramified, Remark 1.5 and Theorems 2.4 and 2.5 imply:

Corollary 2.6. Let $R$ be a Noetherian ring that is locally at each prime ideal analytically unramified and formally equidimensional, and let $I$ be an ideal of $R$. There exists a one-dimensional semilocal extension ring $S$ of $R$ which is essentially of finite type over $R$ and is such that every power of $I$ is contracted from a principal ideal in $S$. If $R$ is an integral domain one can take $S$ to be a domain. Also, there exists a positive integer $k$ such that, for all $n \geq 1$, there exists a primary decomposition $I^{n}=Q_{1} \cap \cdots \cap Q_{s}$ where each $Q_{i}$ contains the $n k$-th power of its radical.

Remark 2.7. In general, an ideal $I$ of a Noetherian integral domain $R$ need not be the contraction of a principal ideal of a birational extension of $R$. For example, if $K$ is a field, $t$ is an indeterminate over $K$, and $R$ is the localization of $K\left[t^{3}, t^{4}, t^{5}\right]$ at the maximal ideal $\left(t^{3}, t^{4}, t^{5}\right) K\left[t^{3}, t^{4}, t^{5}\right]$, then the ideal $I=\left(t^{3}, t^{4}\right) R$ is not the contraction of a principal ideal of a birational extension of $R$.

\section{REFERENCES}

[AM] M. Atiyah and I. Macdonald, Introduction to Commutative Algebra, Addison-Wesley, Reading, MA, 1969. MR 39:4129

[B] M. Brodmann, Asymptotic stability of $\operatorname{Ass}\left(M / I^{n} M\right)$, Proc. Amer. Math. Soc. 74 (1979), 16-18. MR 80c: 13012

[GH] R. Gilmer and W. Heinzer, Ideals contracted from a Noetherian extension ring, J. Pure Appl. Algebra 24 (1982), 123-144. MR 84a:13006 
[HO] W. Heinzer and J. Ohm, Noetherian intersections of integral domains, Trans. Amer. Math. Soc. 167 (1972), 291-308. MR 45:5156

[He] J. Herzog, A homological approach to symbolic powers, Commutative Algebra, Proc. of a Workshop held in Salvador, Brazil, 1988, Lecture Notes in Mathematics 1430, SpringerVerlag, Berlin, 1990, pp. 32-46. MR 91k:13002

[Hu] C. Huneke, Uniform bounds in Noetherian rings, Invent. Math. 107 (1992), 203-223. MR 93b: 13027

[M] H. Matsumura, Commutative ring theory, Cambridge University Press, 1986. MR 88h:13001

[N] M. Nagata, Local rings, Interscience, 1962. MR 27:5790

[Rat] L. J. Ratliff, Jr., On prime divisors of $I^{n}, n$ large, Michigan Math. J. 23 (1976), 337-352. MR 56:15626

[R1] D. Rees, A note on analytically unramified local rings, J. London Math. Soc. 36 (1961), 24-28. MR 23:A3761

[R2] - A note on asymptotically unmixed ideals, Math. Proc. Camb. Phil. Soc. 98 (1985), 33-35. MR 86k:13015

[S1] I. Swanson, Primary decompositions of powers of ideals, Commutative Algebra: Syzygies, Multiplicities, and Birational Algebra: Proceedings of a summer research conference on commutative algebra held July 4-10, 1992 (W. Heinzer, C. Huneke, J.D. Sally, ed.), Contemporary Mathematics, vol. 159, Amer. Math. Soc., Providence, 1994, pp. 367-371. MR 95a:13002

[S2] - Powers of Ideals: Primary decompositions, Artin-Rees lemma and regularity, Math. Annalen (to appear).

[ZS] O. Zariski and P. Samuel, Commutative algebra, Vol. I, Springer, 1975. MR 52:5641

Department of Mathematics, Purdue University, West Lafayette, Indiana 47907-1395

E-mail address: heinzer@math.purdue.edu

Department of Mathematics, New Mexico State University, Las Cruces, New Mexico 88003-8001

E-mail address: iswanson@nmsu.edu 